SCIPP 08/07

\title{
Gauge Mediation with D terms
}

\author{
Linda M. Carpenter, ${ }^{1}$ \\ ${ }^{1}$ Santa Cruz Institute for Particle Physics, \\ Santa Cruz CA 95064
}

\begin{abstract}
I propose implementing General Gauge Mediation using the class of $S U(N) \times U(1)$ SUSY breaking models. As an existence proof, I have utilized the 4-1 model in building multi-parameter gauge mediation. These hidden sectors are relatively easy to use and avoid several model building pitfalls such as runaway directions. In addition models require no special tuning and may produce as many parameters as general gauge mediation allows.
\end{abstract}




\section{Introduction}

Minimal Gauge Mediation (MGM) provides a simple and predictive mass scheme for supersymmetric models [1]. MGM is achieved by adding sets of vector like multiplets to the MSSM which are charged under the normal gauge groups and couple to the hidden sector fields that participate in SUSY breaking. These 'messengers' acquire both a supersymmetric and a nonsupersymmetric mass, and once they are integrated out, these terms give gauginos mass at one loop and scalars mass at two loops. In its simplest incarnation, the messengers are coupled to a hidden sector singlet field that acquires a scalar vev and $\mathrm{F}$ term in the hidden sector,

$$
W=\phi X \bar{X} \rightarrow\langle\phi\rangle X \bar{X}+\theta^{2} F_{\phi} X \bar{X}
$$

where $\mathrm{X}$ are $\mathrm{N}$ messengers and $\phi$ the singlet field. This yields one loop gaugino masses

$$
M_{\lambda i}=\frac{\alpha_{i}}{4 \pi} \frac{F_{\phi}}{\langle\phi\rangle}
$$

The entire mass spectrum is determined by a single parameter $F_{\phi} /\langle\phi\rangle$ and we see that the gaugino and scalar mass ratios are completely fixed.

However, because gauge mediation predicts a spectrum where sparticle masses scale with powers of their gauge couplings, everything that is charged under QCD is very heavy. Thus even though this model is predictive and flavor blind, problems persist in the spectrum. In particular the mass relationship $M_{1}: M_{2}: M_{3} \sim 1: 2: 7$ is predicted for gauginos. The chargino lower mass bound is $105 \mathrm{GeV}$ [2]. We might infer from indirect signal searches like tri-leptons, that the lightest chargino is even heavier, approaching 150 $\mathrm{GeV}$ [3]. In MGM setting the chargino above the mass bound then requires a very heavy gluino due to the fixed gaugino mass ratio. Lower bounds on the lightest charged sparticles of $100 \mathrm{GeV}$ also imply heavy squarks, if minimal gauge mediation holds. Squarks of $700 \mathrm{GeV}$ would induce large corrections to the up type Higgs mass parameter. The conditions for electroweak breaking are known, and Higgs sector parameters must cancel down to the $\mathrm{Z}$ mass. Therefore the amount of tuning needed in the Higgs sector is of order $\left(m_{h_{u}} / m z\right)^{2}$, or sub percent.

A compressed and lighter spectrum would alleviate tuning problems that exist in the Higgs sector and open up SUSY parameter space to new and interesting signals. This would require modifying the gauge mediated predictions for sparticles. Meade et al. have laid out the formalism of General 
Gauge Mediation whereby the gauge mediated spectrum may be determined by up to 6 parameters, including 3 independent parameters in the gaugino sector [4]. Several recent models fall under the category of GGM model building, with weakly coupled renormalizable operators employing chiral fields only. For example Extraordinary GM compresses the spectrum without altering the gaugino mass prediction of MGM [5]. Other proposals compress the spectrum and achieve the full range of GGM parameters [6].

Weakly coupled renormalizable models which change the gaugino mass ratio prediction require, at least, splitting the doublet and triplet messenger couplings and coupling a single set of messengers to multiple scalars. Thus we would have a superpotential like,

$$
W=\left(\lambda_{1} \phi_{1}+\lambda_{2} \phi_{2}+\ldots \lambda_{i} \phi_{i}\right) X \bar{X} \rightarrow \sum \lambda_{q i} \phi_{i} q \bar{q}+\sum \lambda_{l i} \phi_{i} \bar{l}
$$

Now we may make the following field redefinitions:

$$
Z \equiv\left(\sum \lambda_{q i} \phi_{i}\right) ; Y \equiv\left(\sum \lambda_{l i} \phi_{i}\right)
$$

Gaugino masses are now proportional to two scales;

$$
m_{3}=\frac{\alpha_{3}}{4 \pi} \frac{F_{Z}}{\langle z\rangle}, m_{2}=\frac{\alpha_{2}}{4 \pi} \frac{F_{Y}}{\langle y\rangle}, m_{1}=\frac{\alpha_{1}}{4 \pi}\left(\frac{F_{Y}}{\langle y\rangle}+2 / 3 \frac{F_{Z}}{\langle z\rangle}\right)
$$

We see that the gaugino mass ratio of minimal gauge mediation is not preserved and we have achieved a two parameter model.

Models may be complicated even further by adding multiple scalars and multiple messengers. However models like this pose difficult model building challenges. For example, in models with multiple messengers, hypercharge D terms induce one loop masses for scalars proportional to their hypercharge unless an interchange symmetry of the messengers can be made to appear in the low energy theory. In models with multiple scalars which are built purely out of chiral fields, some care is required to make sure the theory is stabilized far from runaway directions so that all fields acquire proper vevs. In addition there is a generic problem with phases. In minimal gauge mediation, gaugino and scalar masses all come from a single mass scale, there are no relative phases between the gaugino masses. However, when model building with multiple scalars and messengers, splitting the gaugino mass requires the addition of many new couplings and in general phases occur.

Instead, I propose the introduction of a single new source of SUSY breaking from a hidden sector $U(1)$. This generates a new operator in the theory, 
a non-supersymmetric mass term which can be added to alter the minimal gauge mediation prediction without multiple scalars or multiple messengers. The GGM parameter counting is distinctly different from models in [6]. In addition, the hidden sector dynamics can be implemented in simple and familiar $S U(N) \times U(1)$ models. In Section 2 I introduce the D-term operator and use it to build the simplest GGM model. In section 3 I review the dynamics of the 4-1 hidden sector. In section $4 \mathrm{I}$ use other operators in the 4-1 model to build gauge mediation and make an attempt at a unified model without phases. Section 5 concludes.

\section{SUSY Breaking D terms}

In addition to $\mathrm{F}$ terms in the hidden sector, we may consider another source of SUSY breaking, a U(1) gauge field whose $D$ term acquires a vev by some dynamical mechanism. Since we want a $D$ term that is the same size as the overall SUSY breaking scale, we may deduce that the $D$ term vev is itself closely connected to, even required for, supersymmetry breaking. The lowest dimension new operator that one may write down with all indices contracted has the form

$$
\frac{c}{M^{2}} \int d^{2} \theta W^{\prime} W^{\prime} X \bar{X}
$$

where $\mathrm{X}$ is matter in a vector-like representation. When the $D$ term is set to its vev this term becomes

$$
c \frac{D^{2}}{M^{2}} x \bar{x}
$$

This is an additional $B$ term, which is a source for nonsupersymmetric masses. Such a term has been used as a source for SUSY breaking messenger masses for example in [7]. The new operator only adds one more parameter to the low energy theory, the scale $\sqrt{c} D / M$, so we may maintain an economy of parameters.

Scalar masses for squarks and sleptons cannot be generated through direct contact terms with the hidden sector gauge field. Holomorphy prevents us from writing such a term in the superpotential. Instead the lowest dimension mass term we may write is $\frac{1}{M^{6}} \int d^{4} \theta W^{\prime} W^{\prime} W^{\prime} \dagger W^{\prime \dagger} Q Q^{\dagger}$, which is highly suppressed and not generated by any divergent diagrams. 


\subsection{A Simple Way To Use D-terms}

Consider a messenger superpotential with a single scalar field Z that gets an F term and a scalar vev, and a hidden sector U(1) field.

$$
W=f Z+y_{Q} Z Q \bar{Q}+y_{L} Z \bar{l}+\frac{\lambda_{Q}}{M^{2}} W^{\prime} W^{\prime} Q \bar{Q}+\frac{\lambda_{L}}{M^{2}} W^{\prime} W^{\prime} \bar{l}
$$

where the couplings for doublet and triplet messengers have been split. Couplings between scalars fields and the extra gauge fields may be forbidden by R symmetry.

$\mathrm{Z}$ gets an $\mathrm{F}$ term and a scalar vev $Z=\langle z\rangle+\theta^{2} F_{z}$. Messengers get a SUSY breaking mass from the $\mathrm{F}$ term and the extra $\mathrm{D}$ term vev. Define $B=D^{2} / M$ so the gaugino masses are

$$
m_{3}=\frac{\alpha_{3}}{4 \pi}\left(\frac{F_{z}}{z}+\frac{\lambda_{Q} B}{y_{Q} z}\right), m_{2}=\frac{\alpha_{1}}{4 \pi}\left(\frac{F_{z}}{z}+\frac{\lambda_{L} B}{y_{L} z}\right)
$$

The $\mathrm{B}$ term may be chosen to be of the same order as $F_{z}$. If the ratio of couplings $\lambda_{Q} / y_{Q}$ is smaller than the ratio of $\lambda_{L} / y_{L}$ we lower the mass ratio of gluinos to the other MSSM gauginos. Notice that there are three distinct parameters $F_{z} / z, \lambda_{Q} B / y_{Q} z$, and $\lambda_{L} B / y_{L} z$.

\section{The $4-1$ Model}

We now must address the best way to achieve a D-term vev. To get a D term of sufficient size, comparable to the overall scale of SUSY breaking, we may build a model in which the $U(1)$ is required for supersymmetry breaking. The '4-1' Model of Dine and Nelson is a simple and interesting example [8].

The model has an $S U(4) \times U(1)$ gauge group. The matter content is as follows (subscripts indicate $U(1)$ charges): an antisymmetric tensor $A_{2}$, a fundamental $F_{-3}$, an anti-fundamental $\bar{F}_{-1}$ and a singlet $S_{4}$. There is only one allowed superpotential term,

$$
W=\lambda S_{4} F_{-3} \bar{F}_{-1}
$$

$S U(4)$ then confines and the gauginos condense generating a non-perturbative

term in the superpotential,

$$
W=\lambda S_{4} F_{-3} \bar{F}_{-1}+\frac{\Lambda_{4}^{5}}{\left(\bar{F}_{i} F^{j} A^{i k} A^{l m} \epsilon_{j k l m}\right)^{1 / 2}} .
$$


This model contains a non-anomalous $\mathrm{R}$ symmetry which is broken once the cosmological constant is tuned to zero, and hence a massive $\mathrm{R}$ axion [9]. The scale of SUSY breaking we will assume is high enough that the $\mathrm{R}$ axion is unobservable.

Making the choice,

$$
A_{2}=\left(\begin{array}{ll}
a \sigma_{2} & \\
& a \sigma_{2}
\end{array}\right), F=\bar{F}=\left(\begin{array}{l}
b \\
0 \\
0 \\
0
\end{array}\right), S=c .
$$

With the rescaling, $\phi \rightarrow \frac{\Lambda}{\lambda^{1 / 5}} \phi$, the D-term is

$$
D_{1}=g_{1} \frac{\Lambda^{2}}{\lambda^{2 / 5}}\left(2|a|^{2}-4|b|^{2}+4|c|^{2}\right)
$$

the scalar potential F-term contribution is,

$$
V_{F}=\lambda^{6 / 5} \Lambda^{4}\left(|b|^{4}+\left|2 b c-\frac{1}{a b^{2}}\right|^{2}+\left|\frac{1}{a^{2} b}\right|^{2}\right)
$$

We may now minimize the potential. Notice that without the D-term there is a runaway direction.

We may take $b \sim \epsilon$ for $\epsilon$ arbitrarily small while $a \sim 1 / \epsilon$ and $c \sim 1 / \epsilon^{2}$. Here we can solve all of the $\mathrm{F}$ term equations. As we go out in the runaway direction SUSY is restored. However, as we turn on the coupling $g_{1}$ we find we can no longer satisfy the F and D term equations and SUSY is broken everywhere. To avoid running away to a supersymmetric minimum, we must generate a $\mathrm{D}$ term. The term $D^{2}$ is quartic in fields and for very small $g_{1}$ the minimum is far from the origin. Because of quartic behavior, as we turn $g_{1}$ up, the minimum moves in closer to zero and the $\mathrm{D}$ term becomes small compared to the F term. Note that The F term is always larger than the D term but regions of parameter space exist, for $\lambda \sim 10 g_{1}$, where they are of the same order. We will see later how the size of this ratio effects phenomenology.

In addition to generating a $\mathrm{D}$ term for the $\mathrm{U}(1)$, the $4-1$ model also gives an additional useful operator for model building, the gaugino condensate of the $S U(4)$ gauge multiplet. 


\section{The Gaugino Condensate}

We see that the in the 4- 1 model, in addition to having a $U(1) \mathrm{D}$ term, there is also a gaugino condensate. Gaugino condensates are useful for generating $\mu$ terms, see for example [10]. Proceeding in a way similar to the previous section, we see that we can couple messengers to the gaugino condensate as well as to the D terms. We write the messenger superpotential

$$
W=y_{1} \frac{W_{1} W_{1}}{M^{2}} X \bar{X}+y_{4} \frac{W_{4} W_{4}}{M^{2}} X \bar{X}
$$

There is now a B-term for the scalar messengers as well as mu term generated by gaugino condensation.

$$
B=y_{1} D^{2} / M^{2} ; \mu=y_{4} \Lambda^{3} / M^{2}
$$

We have built the operators needed for gauge mediation not out $\mathrm{F}$ terms and vevs of chiral fields, but from gauge D terms and gaugino condensates. Gaugino masses are proportional to the ratio of $\mathrm{B}$ and $\mu$

$$
M_{\lambda} \sim y_{1} D^{2} / y_{4} \Lambda^{3}
$$

and are not dependent on the scale M. This simple model does not break the gaugino mass ratio prediction of MGM, but instead reproduces the minimal gauge mediated phenomenology. Achieving the multiple parameters of GGM once again requires splitting the messenger couplings. Below, the messenger sector consists of a single set of messengers in the $5, \overline{5}$ representation however one may imagine repeating these steps for multiple sets of messengers in 5 , $\overline{5}$ or $10, \overline{10}$ representations.

$$
W=y_{1} \frac{W_{1} W_{1}}{M^{2}} Q \bar{Q}+y_{4} \frac{W_{4} W_{4}}{M^{2}} Q \bar{Q}+l_{1} \frac{W_{1} W_{1}}{M^{2}} l \bar{l}+l_{4} \frac{W_{4} W_{4}}{M^{2}} l \bar{l}
$$

Writing everything in terms of $\Lambda$ we have the relation

$$
\begin{aligned}
& M_{3}=\frac{\alpha_{i}}{4 \pi} \frac{y_{1} B}{y_{4} \mu}=\frac{\alpha_{i}}{4 \pi} \frac{y_{1} g_{i}^{2} \Lambda\left(2|a|^{2}-4|b|^{2}+4|c|^{2}\right)^{2}}{y_{4} \lambda^{4 / 5}} \\
& M_{2}=\frac{\alpha_{i}}{4 \pi} \frac{l_{1} B}{l_{4} \mu}=\frac{\alpha_{i}}{4 \pi} \frac{y_{1} g_{i}^{2} \Lambda\left(2|a|^{2}-4|b|^{2}+4|c|^{2}\right)^{2}}{y_{4} \lambda^{4 / 5}}
\end{aligned}
$$

In general $y_{1}, y_{4}, l_{1}, l_{4}$ may all be different from each other. What we need to break the MGM gaugino mass prediction is that $y_{1} / y_{4}$ not be equal to $l_{1} / l_{4}$. In order to avoid messenger vevs we must have $B<\mu^{2}$ or 


$$
\frac{g_{1}^{2}}{\lambda^{4 / 5}}\left(2|a|^{2}-4|b|^{2}+4|c|^{2}\right)^{2}<\Lambda^{2} / M^{2}
$$

For the correct spectrum we may pick point like $\Lambda \sim 10^{8}$, $\mathrm{M} \sim 20 \Lambda$ with couplings, $\lambda=2.6 \times 10^{-2}$ and $g_{1}=6 \times 10^{-1}$ and y's and l's of order $10^{-1}$. We get a spectrum with gauginos in the hundred $\mathrm{GeV}$ range and no vevs for messengers. Since there are two independent parameters for gluinos and winos, we may expect a spectrum with light squarks without the need to tune couplings. This model achieves 2 parameters of the possible 6 of GGM. If we had chosen messengers in a $10, \overline{10}$ representation we would have gotten a three parameter spectrum. In fact, using the 4-1 models, the predictions for number of parameters and low energy spectrum follow from those in [6] where our scales are set by $\mathrm{D}$ terms and gaugino condensates rather that $\mathrm{F}$ terms and vevs of chiral fields.

\subsection{Extra Operators}

We may now attempt to write down potentially dangerous operators that get generated in the Kahler potential. The most important is a coupling of hidden sector fields and messenger fields,

$$
K=\lambda \int d^{4} \theta \frac{F^{\dagger} F X^{\dagger} X}{M^{2}}
$$

This operator does not break $\mathrm{R}$ symmetry and cannot contribute to gaugino masses. However it will induce an operator which is another source for scalar masses, and has been well studied in [11].

This is an extra mass term for messengers; and since messengers only couple to MSSM fields with SM gauge couplings, this new contribution will yield flavor blind masses. However these are not the standard mass terms of minimal gauge meditation. The scalar mass contribution from the new operators is

$$
m_{i}^{2}=-f \sum_{a} \frac{g_{a}^{4}}{128 \pi^{4}} S_{Q} C_{a i} \operatorname{Str} M_{m e s s}^{2} \log \left(\frac{M^{2}}{\Lambda^{2}}\right)
$$

where $\mathrm{S}$ is the Dynkin index of the messengers and $C_{a i}$ is the Casimir for the

scalars. Notice that unlike the standard GM contribution, there is running from the scale of the cut off $M$, presumably where we have integrated out 
some heavy fields to generate the operators $W W X \bar{X}$, to the scale at which the 4-1 model gauginos condense hence the log factor. This is scaled by powers of this operators anomalous dimension. In addition, this operator will be down by a factor of $\alpha_{4}(M)$ compared to the standard GM scalar mass contribution since this operator involves two insertions of the hidden sector $\mathrm{F}$ terms. As long as the $\mathrm{F}$ terms are of manageable size and appear with a reasonable coefficient, we expect this operator not to dominate or drastically alter the spectrum. However, if the F terms become large and the log does not scale away with large negative anomalous dimensions this contribution can become as important as the standard GM contribution to scalar masses or even dominant. If the sign of the operator is negative the spectrum may even become tachyonic. The trick then is to stay in regions of parameter space where $\mathrm{F}$ terms are not too large. Another way around this constraint would be to forbid such operators all together. For example, if the hidden sector fields were sequestered from MSSM fields using boundary conditions in 5-D, these extra contributions may be extremely suppressed.

\subsection{A Unified Model}

In minimal gauge mediation we avoid relative phases in the gaugino sector because all gaugino masses come from a single mass parameter. However models with split gaugino masses usually have a relative phase. We would like as few phases as possible. In addition we would like to make a model that is as simple as possible. We might try to begin with a unified $\mathrm{SU}(5)$, then break it to $S U(4) \times U(1)$ needed for 4-1 SUSY breaking. A 10 and one $\overline{5}$ of $S U(5)$ provide all of the chiral fields needed for the 4-1 model. In general we would begin with different couplings of the $W$ 's to doublet and triplet messengers. The operators $W W X \bar{X}$ are generated by integrating out fields carrying quantum numbers under $S U(4)$ and $U(1)$. If the gauge fields are unified at some high energy we expect that once SU(5) breaks, the $W_{1}$ and $W_{4}$ messenger couplings will split. However it may be possible that the relative phases between terms-which start off the same when $\mathrm{SU}(5)$ is unifiedremain the same. This depends on the dynamics at the high scale. What follows is an attempt to build a model where $S U(4)$ and $U(1)$ unify.

After some numerical estimates, we find that we may get the correct order of magnitude for the MSSM field masses and avoid tachyonic messenger masses if the gaugino condensation scale $\Lambda$ is only a few decades above the cut-off $M$. 
We may compute the scale at which $\mathrm{SU}(4)$ confines by finding the pole in

$$
g(\mu)=\frac{g(\Lambda)}{1+\frac{b g(\Lambda)^{2} \ln \left(\frac{\Lambda}{\mu}\right)}{8 \pi^{2}}}
$$

where b for $S U(N)$ is $3 N_{c}-N_{f}$ here 8 .

Run this coupling up to the unification scale, and it is the value of the coupling $g_{1}$ at high energy which will then run down.

We see that if we run over two decades, our unified coupling is $g(\Lambda) \sim 1.4$. The difficulty with this scenario is that for running over only a few decades the $\mathrm{U}(1)$ and $\mathrm{SU}(4)$ couplings do not split very much, it is not possible to make $g_{1}$ small. Therefore, the minimum of the potential comes close to the origin and the $\mathrm{F}$ terms are generically much bigger than the $\mathrm{D}$ terms. In the non-unified model one is free to pick smaller values for $g_{1}$ and this was not as great of a concern.

The scalar masses are now dominated by the contribution mentioned in the previous section. Unless the hidden sector fields are sequestered with extra dimensions, or the extra operator has very large negative anomalous dimensions the extra contribution to scalar masses will be of the same order as the standard gauge mediated contribution. If the sign of these contributions is negative, some scalars may become tachyonic. In addition the extra contributions may reintroduce tuning by increasing scalar masses. This is not a concern if a suitable sequestering mechanism can be found. Even before we worry about finding suitably high energy dynamics, the viability this model is in question. Thus model building without gaugino phases requires further study.

\section{Conclusions}

It possible to build simple implementations of GGM by stepping outside the bounds of weakly coupled chiral models. Here I have demonstrated the viability of using the 4-1 SUSY breaking model. However it is likely that a range of $S U(N) \times U(1)$ models may also yield good results. In addition, I have shown the existence of a mixture of F-term and 4-1 style SUSY breaking that has different parameter counting than previous attempts at GGM completions. Hence I have generated new models for which a compressed

SUSY spectrum is possible. Attempts to build models without phases in the 
gaugino sector fare worse. The minimal unified attempt to build models leads to large contributions to scalar masses. The problem of phases therefore is not resolved unless a suitable sequestering mechanism can be implemented. This is a topic for further work.

\section{Acknowledgments}

This work was supported in part by DOE grant number DE-FG03-92ER40689. I would like to thank Tom Banks and Michael Dine for many helpful discussions.

\section{References}

[1] M. Dine, A. E. Nelson and Y. Shirman, Phys. Rev. D 51, 1362 (1995) arXiv:hep-ph/9408384, M. Dine and A. E. Nelson, Phys. Rev. D 48, 1277 (1993) |arXiv:hep-ph/9303230|.

[2] G. Abbiendi et al. [OPAL Collaboration], Eur. Phys. J. C 35, 1 (2004) arXiv:hep-ex/0401026|.

[3] S. Dube, J. Glatzer, S. Somalwar and A. Sood, arXiv:0808.1605 [hep-ph].

[4] P. Meade, N. Seiberg and D. Shih, arXiv:0801.3278 [hep-ph].

[5] C. Cheung, A. L. Fitzpatrick and D. Shih, JHEP 0807, 054 (2008) arXiv:0710.3585 [hep-ph]].

[6] L. M. Carpenter, M. Dine, G. Festuccia and J. D. Mason, arXiv:0805.2944 [hep-ph].

[7] L. M. Carpenter, arXiv:hep-ph/0510406.

[8] M. Dine, A. E. Nelson, Y. Nir and Y. Shirman, Phys. Rev. D 53, 2658 (1996) arXiv:hep-ph/9507378.

[9] J. Bagger, E. Poppitz and L. Randall, Nucl. Phys. B 426, 3 (1994) arXiv:hep-ph/9405345.

[10] M. Dine and J. D. Mason, arXiv:0712.1355 [hep-ph].

[11] E. Poppitz and S. P. Trivedi, Nucl. Phys. Proc. Suppl. 62, 281 (1998) arXiv:hep-ph/9707439]. 